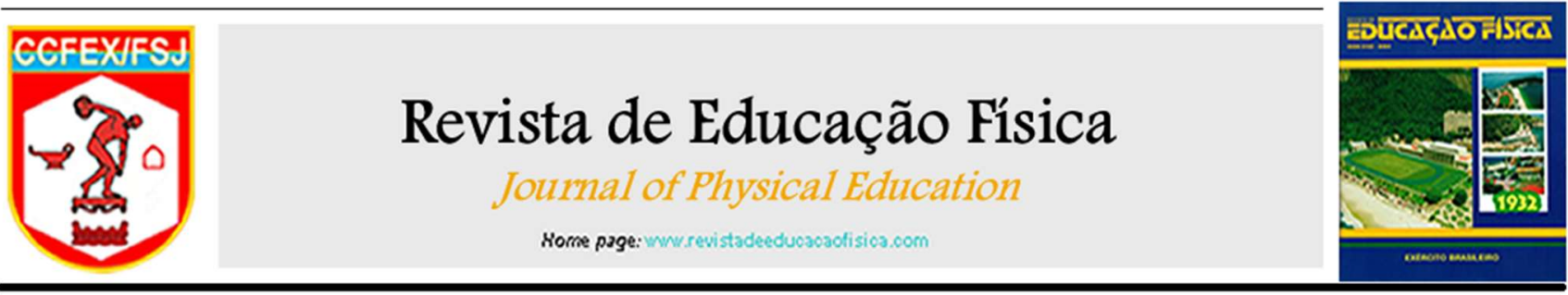

Comentário

Commentary

\title{
Recomendações para a prática de exercício físico para pessoas vivendo com HIV durante a pandemia de COVID-19
}

\section{Physical Exercise for People Living with HIV during the COVID-19 Pandemic}

Fabrízio Di Masi ${ }^{1}$ PhD; Rodrigo Rodrigues da Conceição ${ }^{2}$ PhD; Luiz Claudio Pereira Ribeiro ${ }^{3}$ PhD; Gabriel Costa e Silva ${ }^{1,4} \mathrm{PhD}$

Recebido em: 01 julho de 2020. Aceito em: 08 de julho de 2020

Publicado online em: 28 de julho de 2020.

DOI: $10.37310 /$ ref.v89i1.1628

\section{Resumo}

Introdução: 0 ano de 2020 ficará para sempre marcado na história mundial em função da pandemia da doença causada pelo novo coronavírus (COVID-19) e suas diversas repercussões. Neste contexto, manter níveis regulares de exercício físico durante o período da pandemia pode melhorar a defesa imunológica, auxiliando corpo e mente. A imunidade é um fator de proteção importante contra a COVID-19, especialmente, em grupos que potencialmente possuem maior risco, como por exemplo, as pessoas vivendo com HIV (PVHIV). Entretanto, em função das grandes restrições sociais impostas por diferentes governos, a prescrição e realização de exercícios físicos sem sair de casa tornou-se um grande desafio. 0 isolamento social pode aumentar drasticamente os sentimentos de exclusão e solidão durante o período da pandemia, podendo agravar o estado de saúde das PVHIV que já vivem com estigma e preconceito.

Objetivo: Este comentário se propõe a oferecer informações e recomendações práticas acerca dos exercícios físicos para PVHIV, durante a pandemia da COVID-19.

Conclusão: Destaca-se a importância das PVHIV permanecerem fisicamente ativas durante a pandemia da COVID-19, mantendo um bom nível de condicionamento físico capaz de fortalecer o sistema imunológico, sem deixar de tomar cuidados especiais para que tais exercícios não sejam realizados com sobrecarga exagerada, a fim de promover a saúde e de se evitarem efeitos negativos sobre sua saúde e qualidade de vida., sendo assim prejudiciais em alguma medida.

Palavras-chave: Aids, vírus, SARS-CoV-2, atividade física.
Pontos-Chave Destaque

- Pessoas vivendo com HIV

(PVHIV) devem permanecer

fisicamente ativas durante a pandemia de COVID-19.

- Evitar exercícios de alta intensidade.

- Exercitar-se em locais

privados.

\begin{abstract}
Introduction: The 2020's year will be perpetuated in world history due to the pandemic disease caused by a new coronavirus (COVID-19) and its various repercussions. Maintaining regular physical exercise during the pandemic may improve immune defense, assisting body and mind health. The immunity level is a protective factor against COVID-19, it is extremely important to be aware of groups that are potentially at higher risk, such as people living with HIV (PLWH) who already live with stigma and prejudice. However, due to great social restrictions
\end{abstract}

\footnotetext{
$\S$ Autor correspondente: Fabrízio Di Masi - e-mail: fabdimasi@gmail.com

Afiliações: ${ }^{1}$ Laboratório de Fisiologia e Desempenho Humano (LFDH), Universidade Federal Rural do Rio de Janeiro (UFRRJ); 2Laboratório de Endocrinologia Molecular e Translacional, Universidade Federal de São Paulo (UNIFESP); ${ }^{3}$ Laboratório de Pesquisa em Imunologia e AIDS (LAPIA), Universidade Federal do Estado do Rio de Janeiro (UNIRIO); ${ }^{4}$ Laboratório de Ciência do Movimento Humano, Colégio Pedro II (CP2).
} 
imposed by different governments around the world, the practice of physical exercise without leaving home has become a major challenge, both to prescribe and to perform. The social isolation can drastically increase feelings of exclusion and loneliness during the COVID-19 pandemic period, aggravating the health status of PLWH. Objective: The present text offer information and practical recommendations about physical exercises for PLWH during the pandemic of COVID-19.

Conclusion: We would like to highlight the importance of PLWH, to remain physically active during the COVID-19 pandemic, maintaining a good level of physical fitness capable of improving the responses of the immune system, while taking special care to not promote exaggerated overload, and influence the health and quality of life.

\section{Recomendações para a prática de exercício físico para pessoas vivendo com HIV durante a pandemia de COVID-19}

O ano de 2020 ficará para sempre marcado na história mundial em função da pandemia da doença causada por um novo coronavírus e suas diversas repercussões. O vírus, que teve disseminação iniciada no final de 2019, em Wuhan, na China, provoca doença capaz de gerar graves problemas respiratórios (Severe Acute Respiratory Syndrome: SARS-Cov2), amplamente conhecida como COVID-19(1). A infecção por COVID-19, pode apresentar como uma doença sem grandes complicações do trato respiratório, podendo evoluir para pneumonia grave, falência múltipla de órgãos e consequente óbito, especialmente, em função da imunidade de cada indivíduo. A literatura mostra que pacientes idosos e aqueles que possuem níveis imunológicos reduzidos possuem maior risco de morte, compondo o grupo de pessoas de maior risco para o agravamento da evolução da doença(2).

Além de nutrição(3), prevenção e terapia adequadas(4), o nível de condicionamento físico(5) pode ser imunologicamente importante para enfrentar o tratamento da COVID-19 com sucesso, podendo amenizar os sintomas da doença. É mister ressaltar que o presente texto não se trata de uma recomendação para infectados por COVID-19 como forma de tratamento. Antes, pretende-se ressaltar os benefícios potenciais da manutenção da prática regular de exercícios físicos durante a pandemia, contribuindo para manter o corpo e a mente saudáveis e, assim, melhorar as defesas imunológicas. Ainda existem muitas dúvidas e lacunas na relação da COVID-19 em pessoas vivendo com human immuno-deficiency vírus (HIV): PVHIV $(6,7)$. $\mathrm{O}$ estudo de Zhao et al.(7), reportou um caso de infecção por COVID-19 em paciente HIVpositivo sem que ocorressem alterações detectáveis no RNA. Não obstante, os autores demonstraram que a insuficiência imunológica causada pelo HIV pode atrasar as respostas dos anticorpos. Além disso, Segundo Marziali et al.(9), o isolamento social pode aumentar drasticamente os sentimento de isolamento e solidão no período da pandemia de COVID-19. Da mesma forma, para PVHIV o isolamento social parece agravar bastante seu estado de saúde que, em geral, já vivem em função dos cuidados especiais da doença, estigma e preconceito(8).

Considerando a imunidade um fator para proteção contra a COVID-19, é extremamente importante estar atento aos grupos que potencialmente possuem maior risco, como as PVHIV. Além das terapias antirretrovirais (TARV), a literatura é consistente quanto à importância das atividades e exercícios físicos na melhora da qualidade de vida, dos componentes da aptidão física e manutenção ou aumento número de linfócitos TCD4 em PVHIV(10-12). Entretanto, em função de grandes restrições sociais impostas por diversos governos em todo o mundo, a prática de exercício físico sem sair de casa tornou-se um grande desafio, tanto para prescrevê-los quanto para realizá-los. 
Recentemente, Chen et al.(13) apontou a importância de manter a rotina de exercícios regular durante a pandemia de COVID-19, sem deixar de tomar as precauções e cuidados necessários. Ainsworth(14) ressalta a importância da atividade física na promoção de saúde da população, contudo, não são encontradas na literatura recomendações específicas para PVHIV em isolamento social, sendo caracterizada, então, uma lacuna na literatura científica. Nesse contexto, este comentário se propõe a oferecer recomendações práticas acerca dos exercícios físicos para PVHIV, durante a pandemia da COVID-19.

\section{Recomendações Práticas}

1. Indicadores iniciais para PVHIV iniciarem ou realizarem exercício físico: Em uso da TARV, carga viral indetectável, assintomático e clinicamente estável.

2. Inicialmente, para reduzir $o$ isolamento social, sempre que possível estabeleça vídeo-chamadas virtuais com outros indivíduos para realização das atividades em grupo. Isto pode reduzir a sensação de isolamento e solidão(15).

3. Apesar do efeito crônico oposto, imediatamente após o exercício existe uma baixa de indicadores imunológicos (imunossupressão transitória), principalmente após exercícios intensos(16). Portanto, é importante que PVHIV realizem exercícios em intensidades leves e moderadas. O uso da percepção subjetiva de esforço para controlar a intensidade pode ser uma estratégia interessante(17). De acordo com ACSM(18) a intensidade moderada deve ficar entre 5 a 6 na escala CR-10 de Borg (19).

4. Em PVHIV previamente treinadas, o exercício aeróbico deve ser realizado com duração máxima de 41-50 minutos por sessão(20). Para os destreinados, recomendase um volume entre 10-20 minutos diários, progredindo gradualmente (5-10 minutos a cada 2 semanas) até chegar nos 41-50 minutos por sessão.

5. Procurar opções criativas, como exemplo do uso de bandas elásticas(21) e exercícios funcionais usando o peso do próprio corpo (no caso de exercícios resistidos) e incrementar progressivamente 5$10 \%$ na carga do treino a cada 2 semanas (11).

6. A combinação dos exercícios resistidos com aeróbios pode ser uma estratégia interessante(22).

7. Evitar exercícios muito elaborados que não fazem parte da rotina de exercícios. Manter nutrição e hidratação adequada antes, durante e após as sessões de exercício físico.

8. Realizar exercícios físicos ao menos 3 vezes por semana(23) em dia nãoconsecutivos, podendo chegar a 5 dias semanais no caso de indivíduos fisicamente ativos.

9. Atentar às questões sanitárias de higienização das mãos, equipamentos, ambiente e demais partes do corpo, antes e após cada sessão de exercícios físicos(24).

10. Realizar exercícios físicos em ambiente privado, com boa ventilação e equipamento próprio e de uso individual, buscando exposição ao sol sempre que possível(13, 25-27).

11. Nos locais onde as autoridades sanitárias liberarem as atividades físicas ao ar livre, as PVHIV podem se exercitar evitando aglomerações, respeitando o distanciamento social e seguindo protocolos de higienização.

\section{Conclusão}

Em sumário, gostaríamos de destacar a importância de PVHIV permanecerem fisicamente ativos durante a pandemia da COVID-19, mantendo um bom nível de condicionamento físico capaz de melhorar a respostas do sistema imunológico, tomando os cuidados necessários para evitar sobrecarga exagerada e não influenciar negativamente na saúde e qualidade de vida Dessa forma, o presente texto fornece recomendações para promoção da prática segura de exercícios físicos para PVHIV.

\section{Declaração de conflito de interesses}

Não há nenhum conflito de interesses em relação ao presente estudo.

\section{Declaração de financiamento}

Estudo realizado sem financiamento. 


\section{Referências}

1. World Health Organization. World Health Organization; Geneva: 2020. A coordinated global research roadmap: 2019 novel coronavirus. Available from: https://www.who.int/blueprint/prioritydiseases/keyaction/Coronavirus_Roadmap_V9.pdf?ua $=1$. Accessed 28 Jul 2020 .

2. Wang M, Luo L, Bu H, Xia H. One case of coronavirus disease 2019 (COVID-19) in a patient co-infected by HIV with a low CD4+ T-cell count. International Journal of Infectious Diseases. [Online] 2020;96: 148-150. Available from: doi:10.1016/j.ijid.2020.04.060

3. Jayawardena R, Sooriyaarachchi P, Chourdakis M, Jeewandara C, Ranasinghe P. Enhancing immunity in viral infections, with special emphasis on COVID-19: a review. Diabetes \& Metabolic Syndrome. 2020;14(4): 376-382. DOI: 10.1016/j.dsx.2020.06.009.

4. Eaton LA, Kalichman SC. Social and behavioral health responses to COVID19: lessons learned from four decades of an HIV pandemic. Journal of Behavior Medicine. 2020;43(3): 341-345. Available from: doi:10.1007/s10865-02000157-y

5. Simpson RJ, Kunz H, Agha N, Graff R. Exercise and the regulation of immune functions. Progress in Molecular Biology and Translational Science. 2015;135: 355-380.

DOI: 10.1016/bs.pmbts.2015.08.001.

6. Drain PK, Garrette N. SARS-CoV-2 pandemic expanding in sub-Saharan Africa: considerations for COVID-19 in people living with HIV. EClinicalMedicine. [Online] 2020;22: 100342. Available from: doi:10.1016/j.eclinm.2020.100342.

7. Zhao J, Liao X, Wang H, Wei L, Xing M, Liu L, Zhang Z. Early virus clearance and delayed antibody response in a case of COVID-19 with a history of co-infection with HIV-1 and HCV. Clinical Infectious
Diseases. [Online] 2020; ciaa408. Available from: doi:10.1093/cid/ciaa408.

8. Marziali ME, Card KG, McLinden $\mathrm{T}$, Salters K, Closson K, Wang L, et al. Relationship Between Social Isolation and Mortality Among People Living with HIV in British Columbia, Canada. Canadian Conference on HIV/AIDS Research 2019. May 9-12; Saskatoon, Saskatchewan. https://www.cahr-acrv.ca/wpcontent/uploads/2019/04/CAHR-2019Abstract-Book.pdf Accessed 27 Apr 2020. DOI:.org/10.1007/s10461-020-02872-8.

9. Marziali ME, Card KG, McLinden $\mathrm{T}$, Wang L, Trigg J, Hogg RS. Physical Distancing in COVID-19 May Exacerbate Experiences of Social Isolation among People Living with HIV. AIDS and Behavior. [Online] 2020; s10461-02002872-02878. Available from: doi:10.1007/s 10461-020-02872-8.

10. Di Masi F, Costa e Silva G, Silveira A, Oliveira AJ, Ferry FR, Dantas E. Association between physical activity and quality of live in HIV-positive subjects. Journal of Sports Medicine and Physical Fitness. 2019;59 (3): 538-539. DOI: 10.23736/S0022-4707.18.07754-X.

11. Bessa A, Lopez JC, Di Masi F, Ferry F, Costa e Silva G, Dantas E. Lymphocyte CD4+ cell count, strength improvements, heart rate and body composition on HIVpositive patients during a 3-month strength training program. Journal of Sports Medicine and Physical Fitness. 2017;57(7-8): 1051-1056. DOI: 10.23736/S0022-4707.16.06357-X.

12. Garcia A, Fraga GA, Vieira RCJ, Silva CM, Trombeta JC, Navalta JW, Prestes J, Voltarelli FA. Effects of combined exercise training on immunological, physical and biochemical parameters in individuals with HIV/AIDS. Journal of Sports Sciences. 2014;32(8): 785-792. DOI: $10.1080 / 02640414.2013 .858177$.

13. Chen P, Mao L, Nassis GP, Harmer P, Ainsworth BE, Li F. Wuhan Coronavirus Diasease (COVID-19): The need to maintain regular physical activity while taking precautions. Journal of Sports and 
Health Science. 2020;9(20): 103-104. DOI: $10.1016 /$ j.jshs.2020.02.001.

14. Ainsworth BE. Promoting physical activity in a public health context. Journal of Sports and Health Science. 2018;7(1): 1-2. DOI: $10.1016 /$ j.jshs.2017.10.004.

15. Kay ES, Musgrove K. From HIV to Coronavirus: AIDS Service Organizations Adaptative Responses to COVID-19, Birmingham, Alabama. AIDS and Behavior. [Online] 2020; s10461-02002879-1. Available from: doi:10.1007/s10461-020-02879-1.

16. Papacosta, E, Gleeson M. Effects of intensified training and taper on immune function. Revista Brasileira de Educação Física e Esporte. 2013;27(1) :159-176. //dx.doi.org/10.1590/S180755092013005000001.

17. Morishita S, Tsubaki A, Nakamura $M$, Nashimoto S, Fu JB, Onishi H. Rating of perceived exertion on resistance training in elderly subjects. Expert Review of Cardiovascular Therapy. 2019;17(2): 145-132. doi: 10.1080/14779072.2019.1561278.

18. American College of Sports Medicine. ACSM's Guidelines for Exercise Testing and Prescription. 10th ed. Philadelphia: LWW; 2017. 480 p.

19. Borg G. Borg's Perceived Exertion and Pain Scales. $1^{\text {st }}$ ed. Champaign, IL: Human Kinetics; 1998. 104 p.

20. Kamitani E, Sipe TA, Higa DH, Mullins MM, Soares J. Evaluating the effectiveness of physical exercise interventions in persons living with HIV: overview of systematic reviews. AIDS Education and Prevention. 2017;29(4): 347-363. 10.1521/aeap.2017.29.4.347.

21. Uchida MC, Nishida MM, Sampaio RA, Moritani T, Arai H. Thera-band( ( $)$ ) elastic band tension: references values for physical activity. Journal of Physical Therapy Science. 2016;28(4): 1266-1271. DOI: $10.1589 /$ jpts.28.1266.
22. Olivera VHF, Rosa FT, Santos JC, Wiechmman SL, Narciso AMS, Franzoi de Moraes SM, Webel AR, Deminice R. Effects of a combined exercise training program on health indicators and quality of life of people living with HIV: a randomized clinical trial. AIDS Behaviour. 2020;24(5): 1531-1541. DOI: 10.1007/s10461-019-02678-3.

23. Stringer WW, Berezovskaya M, O'Brien WA, Beck CK, Casaburi R. The effect of exercise training on aerobic fitness, immune indices, and quality of life in $\mathrm{HIV}+$ patients. Medicine and Science in Sports and Exercise. 1998;30(1): 11-16. DOI: $\quad 10.1097 / 00005768-199801000$ 00003.

24. National Strength and Conditioning Association (NSCA). COVID-19 return to training: Guidance on Safe Return to Training For Athletes. Colorado: NSCA; 2020. 4p.

25. Halabchi F, Ahmadinejad Z, Selk-Ghaffari M. COVID-19 Epidemic: Exercise or Not to Exercise; That is the Question! Asian Journal of Sports Medicine. [Online] 2020;11(1). Available from: doi:10.5812/asjsm.102630 [Accessed: 28th July 2020]

26. Jukic I, Calleja-González J, Cos F, Cuzzolin F, Olmo J, Terrados N, Njaradi N, Sassi R, Requena B, Milanovic L, Krakan I, Chatzichristos K, Alcaraz AE . Strategies and Solutions for Team Sports Athletes in Isolation due to COVID-19. Sports (Basel). 2020;8(4): E56. DOI: $10.3390 /$ sports 8040056 .

27. Owens DJ, Allison R, Close GL. Vitamin $\mathrm{D}$ and the Athlete: Current Perspectives and New Challenges. Sports Medicine. 2018;48(S1): S3-S16. DOI: 10.1007/s40279-017-0841-9. 